\title{
Relações de amizade e autoconceito na adolescência: um estudo exploratório em contexto escolar
}

\author{
Relations of friendship and self-concept in adolescence: An exploratory \\ study in the school context
}

\author{
Renato Gomes CARVALHO' \\ Eduardo FERNANDES ${ }^{1}$ \\ Joana CÂMARA ${ }^{1}$ \\ José Alberto GONÇALVES' \\ João ROSÁRIO' \\ Sandra FREITAS ${ }^{1}$ \\ Sandra CARVALHO ${ }^{1}$
}

\begin{abstract}
Resumo
No presente estudo, analisou-se a relação entre a qualidade das relações de amizade e as autoperceções dos adolescentes, expressas no seu autoconceito. A amostra foi constituída por 65 estudantes portugueses, com idades compreendidas entre os 12 e os 16 anos $(M=14)$. Os instrumentos foram o Questionário de Qualidade da Amizade e a versão portuguesa do Perfil de Autoperceção da Escala de Autoconceito para Adolescentes. Através de análises correlacionais, verificou-se uma relação entre a qualidade das relações de amizade e o autoconceito, sendo a dimensão companheirismo e recreação preditora do autoconceito global. Verificou-se, ainda, a ausência de um efeito de gênero na qualidade reportada das relações de amizade. Pelo contrário, ocorreu um efeito moderado do gênero no autoconceito, nomeadamente nas dimensões competência escolar, competência atlética, aparência física e autoestima, com o gênero masculino apresentando resultados médios superiores. Os resultados foram discutidos tendo em conta a importância das relações sociais para o desenvolvimento na adolescência.
\end{abstract}

Palavras-chave: Adolescência; Amizade; Autoconceito; Escolas; Identidade.

\begin{abstract}
In the present study, the relationship between friendship quality and adolescents' self-perceptions, expressed through their self-concept, in the school context was investigated. The sample consisted of 65 Portuguese students aged 12-16 years (Mean = 14). The Portuguese versions of the Friendship Quality Questionnaire and the Portuguese version of the Self-perception Profile of the Adolescent Self-Concept Scale were used. Correlational analyses were carried out and showed an association between friendship quality and adolescents' self-concept. The dimension companionship and recreation was predictive of global self-concept. There was no effect of gender on the friendship quality reported. On the other hand, a moderate effect of gender on self-concept was observed, namely on the following dimensions: academic
\end{abstract}

${ }^{1}$ Universidade da Madeira, Faculdade de Artes e Humanidades. Campus Universitário da Penteada, 9020-105, Funchal, Portugal. Correspondência para/Correspondence to: R.G. CARVALHO. E-mail: <renatoggc@gmail.com>. 
competence, sports competence, physical attractiveness, and self-esteem. In all cases, males had higher scores. The results were discussed considering the importance of social relationships for adolescent development.

Keywords: Adolescence; Friendship; Self-concept; Schools; Identity.

A transição progressiva da esfera de proteção dos pais e da família para uma integração em grupos de pares e na sociedade, que caracteriza a adolescência (Nurmi, 2004), faz com que a análise do modo como se constróem e vivenciam as relações de amizade seja nuclear para a compreensão do percurso de individuação. A qualidade das relações estabelecidas entre cada adolescente e o seu grupo de pares encontrase associada ao seu bem-estar psicológico e integração social (Carvalho \& Novo, 2013; Hartup \& Stevens, 1997), à capacidade para lidar com os desafios do desenvolvimento e a representações mais estruturadas sobre si, expressas no autoconceito.

A natureza das relações de amizade que os adolescentes apresentam relaciona-se, assim, não apenas na integração social, mas também na forma como constroem a sua identidade. No quadro da importância do desenvolvimento social nessa fase da vida, no presente estudo exploratório realizado em contexto escolar, foi analisada a relação entre a qualidade da amizade e o autoconceito na adolescência, bem como as diferenças de gênero naquelas dimensões.

\section{A adolescência como período significativo de desenvolvimento social}

$\mathrm{Na}$ adolescência, abrem-se novos espaços de experimentação e de interação em múltiplos contextos de vida, os quais contribuem para que cada sujeito possa se consciencializar da sua singularidade, desenvolver uma ideia de si próprio e antecipar o futuro. Em seu modelo epigenético de desenvolvimento, Erikson (1968) enfatiza a importância de fatores intrapessoais, interpessoais e culturais na formação da identidade, onde o desenvolvimento representa uma síntese de psicológico através da realização de um conjunto de tarefas psicossociais. O autor sustenta que a principal tarefa da adolescência, expressa no estágio "identidade versus confusão de papéis", reside na construção da identidade, sendo que as relações estabelecidas no seio de um grupo são preponderantes para aquela tarefa. Com efeito, o grupo de pares corresponde a um laboratório social, no qual o adolescente experimenta funções e papéis, toma decisões sem a intervenção dos seus pais e adequa os seus comportamentos às normas socioculturais (Rabaglietti \& Ciairano, 2008). É através do desenvolvimento de relações marcadas pela amizade que cada adolescente caminha em direção à autonomia e adquire um sentido de reciprocidade e cooperação (Simões, 1997).

A análise das relações de amizade pressupõe uma variedade de critérios, nomeadamente a presença ou ausência de uma relação de amizade, a qualidade da mesma (atributos e valências), as características do amigo e as do adolescente, o contexto da amizade e as interações entre a díade (Bacalhau, 2003; Capelinha, 2013; Hartup \& Stevens, 1997; Rabaglietti \& Ciairano, 2008). O critério qualidade da amizade permite explorar as perceções do adolescente a respeito das suas amizades, bem como identificar as particularidades dessa relação e os respectivos recursos existentes ou ausentes na mesma (Freitas, Santos, Correia, Ribeiro, \& Fernandes, 2013). Dessa forma, a qualidade das relações de amizade permite avaliar os polos positivo e negativo, dado que, por um lado, uma amizade de qualidade superior é marcada por atributos de caráter positivo, como a lealdade, a confiança, a reciprocidade, o companheirismo, a confiança e o suporte emocional, e, por outro, uma de qualidade inferior apresenta atributos como a rivalidade, o conflito, a dominância e a crítica (Freitas, 2014; Guimarães, 2012). 
A capacidade de construção e manutenção de relações de amizade satisfatórias constitui-se como um indicador fidedigno da saúde física e mental do adolescente (Carvalho \& Novo, 2013; Hartup \& Stevens, 1997; Rabaglietti \& Ciairano, 2008), além de ser um fator psicossocial protetor, estando associado a níveis superiores de autoestima, autoeficácia e bem-estar (Freitas, 2014). Esses efeitos também se expressam na vida adulta em diferentes vertentes, nomeadamente a acadêmica, a laboral e a social (Hartup \& Stevens, 1997; Sprinthall \& Collins, 2008). A literatura revela, ainda, uma associação entre relações de amizade satisfatórias e maior capacidade de regulação emocional e autonomia, envolvimento na escola, comportamentos pró-sociais, bem como autoperceções mais positivas em determinadas dimensões do autoconceito (Fujimoto \& Valente, 2012; Glick \& Rose, 2011; Marsh, Allen, Ho, Porter, \& McFarland, 2006; Peixoto, 2003; Sprinthall \& Collins, 2008). Com efeito, Gouveia-Pereira, Pedro, Amaral, Alves-Martins e Peixoto (2000) sublinham que as amizades percecionadas como positivas contribuem para a consolidação de um autoconceito bem desenvolvido e vice-versa (Capelinha, 2013).

\section{Relações de amizade e autoconceito na adolescência}

O autoconceito corresponde a um conjunto de representações descritivas, avaliativas e autorreguladoras que se associam àquilo que cada indivíduo sente sobre si, tendo implicações na forma como orienta a sua conduta. O autoconceito é, nesse sentido, um preditor significativo do comportamento e da adaptação dos indivíduos em diferentes contextos (Faria, 2005). Emídio, Santos, Maia, Monteiro e Veríssimo (2008) relevam a necessidade de ele ser perspectivado como entidade cognitiva e psicológica que reflete as perceções de autoeficácia do indivíduo em vários domínios e que remete para avaliações assentes na experiência pessoal e na comparação social
(Neves \& Faria, 2009). O autoconceito pode ser expresso em várias dimensões, nomeadamente acadêmica (autopercepção relativamente ao percurso acadêmico), social (autopercepção das relações interpessoais e da adaptação ao contexto sociocultural), pessoal (autopercepção enquanto pessoa), emocional (autoperceção e autorregulação das emoções), familiar (autopercepção do sujeito como parte integrante da atmosfera familiar) e global (autoavaliação holística do sujeito resultante da análise de todas as dimensões constituintes) (Guimarães, 2012; Peixoto \& Almeida, 2011).

Assim, o autoconceito desenvolve-se com base nas experiências em diversos contextos de vida, incluindo a família, a escola ou o grupo de pares, bem como nas interpretações que os indivíduos fazem dessas experiências e nas avaliações que pessoas significativas fazem dos seus comportamentos (Faria, 2005). No âmbito da influência das experiências entre cada adolescente e os seus pares no autoconceito (Cordeiro, 2006; Fontaine \& Antunes, 2002), verifica-se que o suporte social se encontra associado a autoperceções positivas e níveis superiores de bem-estar (Guimarães, 2012). Por outro lado, os adolescentes que apresentam autoperceções negativas estão mais predispostos a manifestar comportamentos desviantes e trajetórias inadaptativas, o que mitiga a oportunidade de desenvolverem as suas competências sociais e relacionais (Freitas, 2014; Peixoto, 2003; Peixoto \& Almeida, 2011; Sprinthall \& Collins, 2008).

A literatura corrobora, ainda, a ideia de que as relações estabelecidas entre cada adolescente e os seus amigos influenciam dimensões específicas do autoconceito, como, por exemplo, as suas autoperceções relacionadas com a aceitação social (Capelinha, 2013). Emídio et al. (2008) referem que existe uma relação de interdependência entre a aceitação dos pares e o autoconceito social, uma vez que os indivíduos aceites pelos pares perceccionam as suas relações de amizade de um modo mais positivo e vice-versa.

Considerando a relação entre o autoconceito e a qualidade das relações de amizade expressa 
na literatura, no presente estudo procurou-se verificar a existência dessa relação com uma amostra de adolescentes em contexto escolar. Simultaneamente, foram analisadas potenciais diferenças de gênero na qualidade das relações de amizade e na forma como se expressa autoconceito na adolescência. As hipóteses apresentadas a seguir foram, então, definidas.

Hipótese 1. A qualidade das relações de amizade relaciona-se com o autoconceito, nomeadamente nas dimensões de aceitação social, competência escolar e amizades íntimas (Capelinha, 2013; Steinberg \& Morris, 2001).

Hipótese 2. Existe um efeito do gênero na qualidade das relações de amizade, nomeadamente nas dimensões de partilha e intimidade e conflito e traição. Bacalhau (2003) revela que as adolescentes respondem de forma mais assertiva em situações de conflito, utilizando estratégias mais adequadas de resolução de problemas. O autor também refere que estas são mais empáticas, cooperativas, dedicam mais tempo às interações sociais e estão mais predispostas a procurar apoio instrumental e emocional em virtude de apresentarem relações de amizade pautadas pela intimidade, afeto, validação, segurança e proximidade. Esses resultados são corroborados na literatura internacional, onde é referido que o gênero feminino apresenta maiores expetativas de comunidade, intimidade e reciprocidade nas relações de amizade (Hall, 2011; Watson, 2012). Por outro lado, os rapazes valorizam o papel do estatuto e atratividade nessas relações (Hall, 2011; Watson, 2012), têm maior probabilidade de adotar uma postura dominante e agressiva em uma situação conflitual e recorrem mais frequentemente à violência como estratégia de resolução do conflito (Simões, 1997).

Hipótese 3. Existe um efeito do gênero no autoconceito, designadamente na competência atlética e na autoestima, como revela a literatura em contexto português e em outras culturas (Faria, 2005). Os rapazes apresentam percepções mais que empreendem mais atividades físicas com os seus amigos, como jogos e desportos coletivos, e menos atividades de índole conversacional, estas mais frequentes no gênero feminino (Capelinha, 2013; O’Dea \& Abraham, 1999).

\section{Método}

\section{Participantes}

A amostra foi constituída por 65 estudantes (41 do sexo masculino e 24 do feminino, cerca de $61 \%$ e $39 \%$, respectivamente), com idades compreendidas entre 12 e 16 anos $(M=14, D P=1)$ e que frequentavam o terceiro ciclo do ensino básico (sétimo ao nono ano, com cerca de um terço dos estudantes em cada ano) em uma escola na região da Madeira, Portugal. Na constituição da amostra, não foram identificados efeitos de interação Gênero $x$ Idade, $\chi^{2}(4)=1,21, p=0,877$, nem Gênero $x$ Ano, $\chi^{2}(2)=2,20, p=0,332$.

\section{Instrumentos}

Autoconceito (AC): utilizou-se o perfil de autoperceção da Escala de Autoconceito para Adolescentes (EAA) (Harter, 1993, 2012), que foi adaptada e validada para a população portuguesa por Peixoto, Alves-Martins, Mata e Monteiro (1996). A EAA é uma escala de autorrelato destinada a jovens entre 12 e 18 anos e visa analisar o modo como se autopercebem em vários domínios. A escala dividese em duas subescalas, o perfil de autopercepção, composto por 40 itens, e a escala de importância, composta por 14 itens e que avalia o relevo atribuído a cada dimensão do autoconceito (Fontaine \& Antunes, 2002; Peixoto \& Almeida, 2011). As respostas são possíveis em uma escala de tipo Likert, com quatro pontos, conforme cada item é percebido como verdadeiro ou parcialmente verdadeiro para si.

Neste estudo, foi utilizado o perfil de autopercepção (alfa de Cronbach para a presente 
amostra $=0,90)$, que compreende os seguintes domínios, cada um constituído por seis itens: competência escolar, relativa à perceção de competência cognitiva aplicada ao trabalho na escola $(\alpha=0,80)$; aceitação social, ou seja, saber como fazer novos amigos, lidar com os outros e ser aceito ou ser popular $(\alpha=0,85)$; competência atlética, relativa à capacidade desportiva $(\alpha=0,88)$; aparência física $(\alpha=0,76)$; atração romântica, relativa ao ser ou não atrativo romanticamente para outros $(\alpha=0,79)$; comportamento, isto é, o grau em que a pessoa se comporta da forma como é esperado que se comporte $(\alpha=0,70)$; amizades íntimas, relativa à capacidade de fazer amigos próximos $(\alpha=0,89)$; e autoestima, uma perceção global avaliativa sobre si $(\alpha=0,82)$.

Qualidade da Amizade (QA): recorreu--se ao Questionário de Qualidade da Amizade (Parker \& Asher, 1993), adaptado e validado para a população portuguesa por Freitas, Santos, Correia, Ribeiro e Fernandes (2013), uma escala de autorrelato a qual avalia a percepção que os adolescentes têm a respeito dos diversos aspectos qualitativos da sua melhor amizade em contexto escolar (Capelinha, 2013). Esse questionário é constituído por 41 itens $(\alpha=0,92)$ com uma escala de resposta de tipo Likert entre 1 (nada verdadeiro) e 5 (muito verdadeiro). Para além da medida total, o instrumento é constituído por seis subescalas denominadas validação e cuidado $(\alpha=$ $0,81)$, ajuda e orientação $(\alpha=0,88)$, companheirismo e recreação $(\alpha=0,54)$, partilha e intimidade $(\alpha=$ $0,86)$, resolução de conflito $(\alpha=0,58)$ e conflito e traição $(\alpha=0,78)$.

\section{Procedimentos}

Após a obtenção do consentimento informado dos estudantes e dos seus encarregados de educação, os instrumentos foram aplicados em contexto de sala de aula, em uma sessão com duração de cerca de 45 minutos. 0 acesso às turmas e o agendamento das sessões foram efetuados em concordância com o Conselho Executivo e com os diretores de cada turma. Neste estudo, foram atendidos os aspectos éticos da pesquisa com seres humanos, destacando-se o consentimento livre e informado, a confidencialidade no tratamento dos dados e uma análise preliminar onde foram analisados os potenciais riscos e benefícios da pesquisa.

Para testar a hipótese 1, foram calculados os coeficientes de correlação de Pearson entre os resultados das variáveis relativas à $\mathrm{QA}$ e ao $\mathrm{AC}$. Testou-se, ainda, um modelo stepwise de regressão linear múltipla, de forma a identificar potenciais preditores do AC global a partir das dimensões da QA. Apesar da seleção de variáveis ter sido efetuada através de modelos stepwise, também recorreu-se ao Variance Inflaction Factor (VIF) para identificar potenciais problemas de multicolinariedade. Para testar as hipóteses 2 e 3, foram feitas Análises da Variância Multivariada two way (MANOVA, Multivariate Analysis of Variance) de modo a verificar a existência de um efeito de gênero na QA e no AC. Foi incluída no modelo a variável idade.

\section{Resultados}

Os resultados, patentes na Tabela 1, confirmam parcialmente a hipótese 1 , tendose verificado uma associação moderada entre a qualidade global da amizade e as dimensões competência escolar $(r=-0,318, p<0,01)$ e amizades íntimas do AC $(r=0,327, p<0,01)$. É importante ressaltar que a correlação obtida no caso da competência escolar é negativa. Foi ainda observada uma relação moderada entre a QA e a dimensão atração romântica do $\mathrm{AC}(r=0,336$, $p<0,01)$. Não foi identificada uma associação entre a QA e a dimensão aceitação social do AC. Apesar de não ter sido identificada uma relação entre os resultados relativos à $\mathrm{QA}$ e ao AC global, observou-se uma relação entre este e a dimensão companheirismo e recreação da QA $(r=0,287, p$ $<0,05)$.

Na matriz de correlações obtida, identificase uma associação negativa entre a competência escolar do AC e a resolução de conflitos $(r=-0,291$, 
Tabela 1

Matriz de correlações de Pearson entre as dimensões do autoconceito e a qualidade das amizades. Funchal, Madeira, Portugal, 2015

\begin{tabular}{lccccccc}
\hline Dimensões & QA global & VC & RC & CT & AO & CR & PI \\
\hline AC global & 0,132 & 0,121 & 0,106 & 0,003 & 0,076 & $0,287^{*}$ & 0,003 \\
Competência escolar & $-0,318^{* *}$ & $-0,233^{* *}$ & $-0,291 * *$ & $-0,059$ & $-0,295^{*}$ & 0,103 & $-0,424^{* *}$ \\
Aceitação social & 0,094 & 0,022 & $-0,008$ & 0,153 & 0,068 & 0,232 & $-0,039$ \\
Competência atlética & 0,099 & 0,109 & 0,088 & 0,079 & $-0,003$ & 0,063 & 0,075 \\
Aparência física & $-0,086$ & $-0,118$ & $-0,139$ & 0,035 & $-0,016$ & 0,186 & $-0,233$ \\
Atração romântica & $0,336 * *$ & 0,214 & 0,223 & 0,210 & $0,257 *$ & 0,160 & $0,254 *$ \\
Comportamento & $-0,011$ & 0,132 & 0,073 & $-0,146$ & $-0,043$ & 0,070 & $-0,065$ \\
Amizades íntimas & $0,327 * *$ & 0,219 & 0,171 & $-0,018$ & $0,327 * *$ & $0,350 * *$ & $0,267 * *$ \\
Autoestima & $-0,109$ & $-0,102$ & $-0,044$ & 0,000 & $-0,051$ & 0,191 & $-0,317 *$ \\
\hline
\end{tabular}

Nota: ${ }^{*} p<0,05 ; * * \mathrm{p}<0,01$.

AC: Autoconceito; QA: Qualidade da Amizade; VC: Validade e Cuidado; RC: Resolução do Conflito; CT: Conflitos e Traição; AO: Ajuda e Orientação; CR: Companheirismo e Recreação; PI: Partilha e Intimidade.

Tabela 2

Diferenças de gênero nas dimensões do autoconceito. Funchal, Madeira, Portugal, 2015

\begin{tabular}{lccccccc}
\hline \multirow{2}{*}{ Dimensões do autoconceito } & \multicolumn{2}{c}{ Feminino } & \multicolumn{2}{c}{ Masculino } & & \multirow{2}{*}{$F_{(1,65)}$} & \\
\cline { 2 - 4 } & $M$ & $D P$ & $M$ & $D P$ & & \\
\hline Competência escolar & 2,40 & 0,15 & 2,99 & 0,12 & & $8,91 * *$ & 0,14 \\
Aceitação social & 2,96 & 0,17 & 3,11 & 0,13 & & $0,55^{\text {n.s. }}$ & - \\
Competência atlética & 2,41 & 0,19 & 2,96 & 0,15 & & $5,09 * *$ & 0,09 \\
Aparência física & 2,56 & 0,15 & 2,98 & 0,12 & & $4,78^{*}$ & 0,08 \\
Atração romântica & 2,64 & 0,16 & 2,72 & 0,12 & & $0,16^{\text {n.s. }}$ & - \\
Comportamento & 2,91 & 0,13 & 2,82 & 0,10 & & $0,26^{\text {n.s. }}$ & - \\
Amizades íntimas & 3,35 & 0,20 & 3,27 & 0,16 & & $0,09^{\text {n.s. }}$ & - \\
Autoestima & 2,84 & 0,16 & 3,22 & 0,12 & & $3,59 *$ & 0,06 \\
\hline
\end{tabular}

Nota: ${ }^{*} p<0,05 ;{ }^{*}{ }^{*} p<0,01$. ${ }^{\text {n.s.: }}$ não significativo.

$p<0,05)$, ajuda e orientação $(r=-0,295, p<0,05)$ e partilha e intimidade $(r=-0,424, p<0,01)$ da QA. Por outro lado, a dimensão partilha e intimidade da QA apresenta uma correlação positiva com as dimensões atração romântica $(r=0,254, p<0,05)$ e amizades íntimas $(r=0,267, p<0,05)$ do AC e negativa com a dimensão autoestima $(r=-0,317, p$ $<0,05)$. Ressalta-se, por fim, a associação positiva entre a dimensão ajuda e orientação da QA e as dimensões atração romântica $(r=0,257, p<0,05)$ e amizades íntimas $(r=0,327, p<0,01)$ do $A C$. Companheirismo e recreação encontra-se ainda relacionado com a dimensão amizades íntimas do AC $(r=0,350, p<0,01)$.

Em uma análise que visou identificar dimensões da QA que se constituíssem como preditoras do $A C$ global, os resultados revelam um modelo significativo, $F_{(1,64)}=5,65, p<$ percentagem da variabilidade explicada. A dimensão companheirismo e recreação da QA foi o melhor preditor do AC global na amostra em estudo, $B=0,130, S E=0,055, t(64)=2,38$, $p<0,05$.

Quanto à presença de um efeito de gênero na $\mathrm{QA}$, os resultados revelam que o mesmo não se verificou, $F_{(7,49)}=2,10, p=0,06, \lambda$ de Wilks $=$ 0,77 , o que permite refutar a hipótese 2 . Também não existiu um efeito de interação Gênero x Idade, $F_{(7,65)}=0,86, p=0,67, \lambda$ de Wilks $=0,63$.

Com relação à hipótese 3 , verifica-se um efeito do gênero no $A C, F_{(9,47)}=2,10, p<0,05, \lambda$ de Wilks $=0,71, p^{2}=0,28$, com uma magnitude moderada nas dimensões competência escolar, competência atlética, aparência física e autoestima. Em todos os casos, é o gênero masculino que reporta valores superiores, o que apoia a hipótese 3. Os resultados demonstram a inexistência de um 
efeito da idade, quer isoladamente, $F_{(1,65)}=1,35$, $p=0,106, \lambda$ de Wilks $=0,40$, quer em interação com o gênero, $F(4,65)=1,21, p=0,206, \lambda$ de Wilks $=0,44$.

\section{Discussão}

No presente estudo, forneceu-se uma contribuição para a análise da relação entre a qualidade das relações de amizade (QA) e o autoconceito (AC) na adolescência, a qual não tem sido muito explorada na literatura. Verificou-se, ainda, potenciais efeitos de gênero na qualidade das relações de amizade e nas autoperceções que os adolescentes apresentam. Os resultados confirmaram parcialmente a hipótese 1 de que a QA se encontra relacionada com o AC. No entanto, ao contrário do hipotetizado, as associações significativas ocorreram somente nas dimensões competência escolar e nas amizades íntimas, bem como na dimensão atração romântica, sendo que no primeiro caso a relação é negativa. Por outro lado, verificou-se um padrão mais alargado de correlações entre diversas dimensões da QA e do $A C$, indicando que a forma como ambos se relacionam deverá ser colocada em um plano mais particular, nomeadamente das dimensões específicas que caracterizam aqueles construtos. Nesse sentido, ressalta-se a importância da dimensão companheirismo e recreação das amizades como o melhor preditor do AC global na amostra analisada.

A associação negativa entre a competência escolar e o AC não confirma os resultados da literatura, os quais indicam que as relações de amizade mais estáveis e sólidas estão associadas a autoperceções de competência escolar mais positivas (Capelinha, 2013; Peixoto, 2003), sendo, por isso, necessária maior exploração deste tópico. Face à metodologia utilizada no presente estudo, poder-se-á considerar a potencial existência de um efeito da dimensão e caracterização da amostra (pequena e majoritariamente masculina), bem como da ausência do controle de outras variáveis relevantes, principalmente o desempenho escolar. Assim, um maior investimento dos participantes nas relações de amizade em detrimento da competência acadêmica ou, contrariamente, de centração do desempenho acadêmico e desvalorização das relações interpessoais, poderá ter tido um efeito nos resultados obtidos no presente estudo. Acresce que, tratando-se de uma amostra de jovens adolescentes e que a QA se refere à melhor relação de amizade, aqueles participantes poderiam não ter tido a oportunidade de estabelecer uma relação de amizade mais diferenciada.

Por outro lado, a associação entre a QA e a dimensão amizades íntimas do $A C$, bem como a associação entre esta e algumas dimensões da qualidade das relações de amizade (ajuda e orientação, companheirismo e recreação e partilha e intimidade) valida a perspectiva de que nas amizades de qualidade figuram a entreajuda, a cooperação, a orientação, o companheirismo, a reciprocidade e a intimidade como atributos indutores de uma autopercepção positiva da intimidade presente nas amizades (Bacalhau, 2003; Cordeiro, 2006; Gouveia et al., 2000).

Ao contrário do hipotetizado (hipótese 2), não foi identificado um efeito de gênero na QA. Não obstante a literatura apontar que as amizades no gênero feminino sejam pautadas no diálogo, intimidade, empatia, resolução de conflitos, cooperação e entreajuda (Hall, 2011; McDonald, Malti, Killen, \& Rubin, 2014; Steinberg \& Morris, 2001; Watson, 2012), enquanto as do gênero masculino se expressem na partilha de informações e atividades coletivas, esse padrão não foi verificado no presente estudo. Assim, o enfoque superior no estatuto e no papel que caracteriza as amizades no gênero masculino, e que é influenciado por dimensões mais estruturadas (por exemplo, decorrentes de circunstâncias como o trabalho, o clube, entre outros) (Hall, 2011; Watson, 2012), poderá não se ter feito sentir na presente amostra, dado ser constituída por participantes com idades e em um período do desenvolvimento em que essas dimensões ainda não são muito prevalentes. Além 
disso, poderá também ter existido um efeito da dimensão da amostra e da distribuição não equitativa em termos de gênero, o que pode ter contribuído para a menor probabilidade de identificação de diferenças significativas.

Quanto à influência do gênero no AC, constatou-se a existência de um efeito moderado, onde os participantes do sexo masculino reportam maiores autoestima, competência atlética e escolar e melhor aparência física. Esses resultados permitiram confirmar a hipótese 3, sustentada na literatura e que refere uma tendência do gênero masculino reportar níveis superiores de competência atlética (Capelinha, 2013; Faria, 2005; Peixoto, 2003; Sprinthall \& Collins, 2008). A autoestima superior reportada pelos homens também corrobora os resultados da literatura, que indica maior insegurança e insatisfação do gênero feminino no que diz respeito à aparência física, bem como um defasamento entre a aparência percebida e o valor que the é atribuído (Faria, 2005; Harter, 1993), o que pode estar relacionado à sua autoestima (Capelinha, 2013; Freitas, 2014; Guimarães, 2012).

A apresentação pelo gênero masculino de um AC escolar mais positivo suscita reflexão, já que contraria a literatura, a qual refere que as mulheres têm resultados escolares superiores em diferentes níveis de ensino, mesmo controlando-se variáveis contextuais, como o nível socioeconômico (Carvalho, 2016; D. Voyer \& Voyer, 2014). Ainda que os resultados não permitam clarificar os motivos subjacentes a esse efeito, poder-se-á hipotetizar potenciais efeitos de maior otimismo dos rapazes, que os poderá levar a sobrestimar as suas capacidades. De fato, em um estudo que analisou as influências genéticas e ambientais na variabilidade do otimismo na adolescência, Mavio lu, Bomsma com classificações elevadas, poderá ter influído na sua auto-avaliação acadêmica).

\section{Conclusão}

Considerando a relação entre o autoconceito e o comportamento adaptativo em diversos contextos (Faria, 2005), bem como a diversidade de oportunidades que os contextos escolares podem e devem providenciar aos adolescentes (Carvalho, 2015), os resultados do presente estudo sublinham a importância das experiências que esses sujeitos têm em contexto escolar para o desenvolvimento de uma perceção de competências reais e um autoconceito positivo (Valentini, Villwock, Vieira, Vieira, \& Barbosa, 2010). Se os indivíduos que reconhecem e valorizam a sua competência têm mais probabilidades de a rendibilizarem, também é verdade que os adolescentes os quais subestimam suas capacidades têm expectativas negativas acerca da sua realização futura, evitam a ação e o treino de competências nos vários domínios do desempenho (Faria, 2005), o que poderá ter impactos significativos nas suas trajetórias de desenvolvimento. Sendo as escolas contextos privilegiados de interação e de integração social (Carvalho, 2015), onde os adolescentes têm desafios e oportunidades que contribuem para processos de reflexão e observação simultâneos, essenciais para a construção da identidade (Erikson, 1968), deverão ser promovidos ambientes relacionais em contexto escolar que favoreçam a constituição de redes de amizades e a melhoria da sua qualidade, através de culturas de relação, comunicação e cooperação.

Tendo em conta a importância do desenvolvimento social na adolescência e em um quadro de escassez de referências relativas ao estudo da relação entre as relações de amizade e construção do autoconceito na adolescência, os resultados obtidos encorajam, em suma, o aprofundamento da investigação neste domínio. No entanto, este estudo apresenta algumas limitações, que deverão ser consideradas. Importa referir sua natureza contextualizada, que recorreu a uma amostra de dimensão reduzida, em uma escola e 
com recurso somente a medidas de autorrelato. Para ser possível captar a complexidade das relações entre as amizades dos adolescentes e o seu autoconceito, será relevante, não só reduzir efeitos de contexto, recorrendo a amostras maiores, com maior distribuição geográfica e mais equitativa em termos de gênero, como também recorrer a diferentes metodologias. Estas, além de analisarem outros critérios os quais caracterizam as amizades, mas não foram contemplados no presente estudo (e.g., número, estabilidade e características das relações), deverão ser considerados instrumentos que permitam medidas de auto e heterorrelato e que incluam percepções de outros agentes significativos, como os pais, os professores e os amigos. Deverá recorrer-se ainda a desenhos longitudinais, que analisem o impacto de médio e longo prazo da qualidade das relações de amizade na construção do autoconceito.

A qualidade das relações de amizade apresenta grande relevância para o desenvolvimento na adolescência, já que contribui para a aquisição de conhecimentos e para a interiorização de valores centrais e estruturantes como a confiança, a lealdade, a reciprocidade e a responsabilidade. Além disso, concede aos adolescentes a possibilidade de explorar o mundo social e desenvolver competências sociais, emocionais e de raciocínio moral, bem como de fortalecer o sentimento de autoeficácia e a capacidade de efetuar projeções para o futuro. Considera-se, por isso, que o presente estudo constitui uma contribuição para a exploração necessária de um tema relevante para a compreensão do desenvolvimento na adolescência.

\section{Colaboradores}

Todos os autores participaram na concepção, coleta e análise de dados, redação e revisão do artigo.

\section{Referências}

Bacalhau, A. (2003). Amizade, reciprocidade e qualidade: a relação existe? (Tese de mestrado não-publicada).
Instituto Universitário de Ciências Psicológicas, Sociais e da Vida, Lisboa, Portugal.

Capelinha, J. C. C. (2013). A qualidade das relações de amizade na adolescência e as suas implicações ao nivel do autoconceito e autoestima. (Tese de mestrado não-publicada). Instituto Universitário de Ciências Psicológicas, Sociais e da Vida, Lisboa, Portugal.

Carvalho, R. G. (2015). Future time perspective as a predictor of adolescents' adaptive behavior in school. School Psychology International, 36(5), 482-497. https://doi. org/10.1177/0143034315601167

Carvalho, R. G. (2016). Gender differences in academic achievement: The mediating role of personality. Personality and Individual Differences, 94, 54-58. https:// doi.org/10.1016/j.paid.2016.01.011

Carvalho, R. G., \& Novo, R. F. (2013). Características da personalidade e relacionamento interpessoal na adolescência. Avaliação Psicológica, 12(1), 27-36.

Cordeiro, R. A. (2006). Aparência física e amizade íntima na adolescência: estudo num contexto pré-universitário. Análise Psicológica, 24(4), 509-517. https://doi. org/10.14417/ap.548

Emídio, R., Santos, A. J., Maia, J., Monteiro, L., \& Veríssimo, M. (2008). Auto-conceito e aceitação pelos pares no final do período pré-escolar. Análise Psicológica, 26(3), 491-499. https://doi.org/10.14417/ap.510

Erikson, E. H. (1968). Identity: Youth and crisis. New York: Norton.

Faria, L. (2005). Desenvolvimento do auto-conceito físico nas crianças e nos adolescentes. Análise Psicológica, 4(23), 361-371. https://doi.org/10.14417/ap.554

Fontaine, A. M., \& Antunes, C. (2002). Avaliação do autoconceito e da auto-estima na adolescência: comparação de dois instrumentos. Cadernos de Consulta Psicológica, 17(18), 119-133.

Freitas, M. C. N. (2014). O papel dos melhores amigos e do grupo de pares nas trajectórias de retirada social durante a adolescência (Tese de doutoramento não-publicada). Instituto Universitário de Ciências Psicológicas, Sociais e da Vida, Lisboa, Portugal.

Freitas, M., Santos, A. J., Correia, J., Ribeiro, O., \& Fernandes, E. (2013). Análise factorial confirmatória do modelo do Questionário da Qualidade da Amizade numa amostra de jovens adolescentes Portuguesa. Laboratório de Psicologia, 11(2), 163-175. https://doi. org/10.14417/lp655

Fujimoto, K., \& Valente, T. W. (2012). Decomposing the components of friendship and friends' influence on adolescent drinking and smoking. Journal of Adolescent Health, 51(2), 136-143. https://doi.org/10.1016/j. jadohealth.2011.11.013

Glick, G. C., \& Rose, A. J. (2011). Prospective associations 
between friendship adjustment and social strategies: Friendship as a context for building social skills. Developmental Psychology, 47(4), 1117-1132. https:// doi.org/10.1037/a0023277

Gouveia-Pereira, M., Pedro, I., Amaral, V., Alves-Martins, M., \& Peixoto, F. (2000). Dinâmicas grupais na adolescência. Análise Psicológica, 18(2), 191-201. https://doi.org/10.14417/ap.414

Guimarães, J. V. C. (2012). Autoconceito, autoestima e comportamentos desviantes em adolescentes (Tese de mestrado não-publicada). Instituto Universitário de Ciências Psicológicas, Sociais e da Vida, Lisboa, Portugal.

Hall, J. A. (2011). Sex differences in friendship expectations: A meta-analysis. Journal of Social and Personal Relationships, 28(6), 723-747. https://doi. org/10.1177/0265407510386192

Harter, S. (1993). Self and identity development. In S. S. Feldman \& G. R. Elliott (Eds.), At the threshold. The developing adolescent (pp.352-387). Cambridge: Harvard University Press.

Harter, S. (2012). Self-perception profile for adolescents: Manual and questionnaires. Denver: University of Denver.

Hartup, W. W., \& Stevens, N. (1997). Friendship and adaptation in the life course. Psychological Bulletin, 121(3), 355-370. https://doi.org/10.1037/00332909.121.3.355

Marsh, P., Allen, J. P., Ho, M., Porter, M., \& McFarland, F. C. (2006). The changing nature of adolescent friendships longitudinal links with early adolescent ego development. Journal of Early Adolescence, 26(4), 414-431. https://doi.org/10.1177/0272431606291942

Mavio lu, R. N., Boomsma, D. I., \& Bartels, M. (2015). Causes of individual differences in adolescent optimism: A study in Dutch twins and their siblings. European Child \& Adolescent Psychiatry, 24(11), 1381-1388. https://doi. org/10.1007/s00787-015-0680-x

McDonald, K. L., Malti, T., Killen, M., \& Rubin, K. H. (2014). Best friends' discussions of social dilemmas. Journal of Youth and Adolescence, 43(2), 233-244. https://doi. org/10.1007/s10964-013-9961-1

Neves, S. P., \& Faria, L. (2009). Auto-conceito e autoeficácia: semelhanças, diferenças, inter-relação e influência no rendimento escolar. Revista da Faculdade de Ciências Humanas e Sociais do Porto, 6, 206-218.

Nurmi, J. E. (2004). Socialization and self-development. In R. M. Lerner \& L. Steinberg (Eds.), Handbook of adolescent psychology (2a ed., pp.85-124). Hoboken: Wiley.

O’Dea, J. A., \& Abraham, S. (1999). Association between self-concept and body weight, gender, and pubertal development among male and female adolescents. Adolescence, 34(133), 69-79.

Parker, J. G., \& Asher, S. R. (1993). Friendship and friendship quality in middle childhood: Links with peer group acceptance and feelings of loneliness and social dissatisfaction. Developmental Psychology, 29(4), 611621. https://doi.org/10.1037/0012-1649.29.4.611

Peixoto, F., Alves-Martins, M., Mata, L., \& Monteiro, V. (1996). Adaptação da escala de auto-conceito para adolescentes de Susan Harter para a população portuguesa. Avaliação Psicológica: Formas e Contextos, 4, 531-537.

Peixoto, F. (2003). Auto-estima, autoconceito e dinâmicas relacionais em contexto escolar (Tese de doutorado nãopublicada). Universidade do Minho, Braga, Portugal.

Peixoto, F., \& Almeida, L. S. (2011). A organização do autoconceito: análise da estrutura hierárquica em adolescentes. Psicologia: Reflexão e Crítica, 24(3), 533-541. https://doi.org/10.1590/S010279722011000300014

Rabaglietti, E., \& Ciairano, S. (2008). Quality of friendship relationships and developmental tasks in adolescence. Cognition, Brain, Behavior, 12(2), 183-203.

Simões, C. E. N. (1997). Relações de amizade e processos de grupo em adolescentes. Um estudo específico de quatro turmas de alunos do $9^{\circ}$ ano de escolaridade (Tese de mestrado não-publicada). Instituto Universitário de Ciências Psicológicas, Sociais e da Vida, Lisboa, Portugal.

Sprinthall, N. A., \& Collins, A. (2008). Psicologia do adolescente: uma abordagem desenvolvimentista (4 ${ }^{\text {a }}$ ed.). Lisboa: Fundação Calouste Gulbenkian.

Steinberg, L., \& Morris, A. S. (2001). Adolescent development. Annual Review of Psychology, 52, 83-110. https://doi. org/10.1146/annurev.psych.52.1.83

Valentini, N. D., Villwock, G., Vieira, L. F., Vieira, J. L. L., \& Barbosa, M. L. L. (2010). Validação brasileira da escala de autopercepção de Harter para crianças. Psicologia: Reflexão e Crítica, 23(3), 411-419. https:// doi.org/10.1590/S0102-79722010000300001

Voyer, D., \& Voyer, S. D. (2014). Gender differences in scholastic achievement: A meta-analysis. Psychological Bulletin, 140(4), 1174-1204. https://doi.org/10.1037/ a0036620

Watson, D. C. (2012). Gender differences in gossip and friendship. Sex Roles, 67(2), 494-502. https://doi. org/10.1007/s11199-012-0160-4

Recebido: março 3, 2016

Versão final: junho 10, 2016

Aprovado: agosto 10, 2016 